\title{
Abstract and Effector-Specific Representations of Motor Sequences Identified with PET
}

\author{
Scott T. Grafton, ${ }^{1}$ Eliot Hazeltine, ${ }^{2}$ Richard B. Ivry ${ }^{2}$ \\ ${ }^{1}$ Departments of Neurology and Radiology, Emory University School of Medicine and the Emory Positron Emission \\ Tomography Imaging Center, Atlanta, Georgia 30322, and 'Department of Psychology, University of California, \\ Berkeley, California 94720
}

Positron emission tomography was used to identify neural systems involved in the acquisition and expression of sequential movements produced by different effectors. Subjects were tested on the serial reaction time task under implicit learning conditions. In the initial acquisition phase, subjects responded to the stimuli with keypresses using the four fingers of the right hand. During this phase, the stimuli followed a fixed sequence for one group of subjects (group A) and were randomly selected for another group (group B). In the transfer phase, arm movements were used to press keys on a substantially larger keyboard, and for both groups, the stimuli followed the sequence. Behavioral indices provided clear evidence of learning during the acquisition phase for group $A$ and transfer when switched to the large keyboard. Sequence acquisition was associated with learning-related increases in regional cerebral blood flow $(\mathrm{rCBF})$ in a network of areas in the contralateral left hemisphere,

One important component of human motor learning is the assembly of different movements into sequential action. Sequences of movements can be learned at multiple levels of representation (MacKay, 1982; Cohen et al., 1990; Mayr, 1996; Schmidtke and Heuer, 1996). Functional imaging studies and transcortical magnetic stimulation have begun to distinguish the separate neural systems that are involved in the cognitive, perceptual, motoric, and temporal aspects of learning (Friston et al., 1992; Seitz and Roland, 1992; Jenkins et al., 1994; Pascual-Leone et al., 1994; Berns et al., 1997; Shadmehr and Holcomb, 1997). We have examined the neural systems associated with implicit sequence learning using the serial reaction time task (SRT). In this task, one of four stimuli is presented on each trial, and the subjects press the response key mapped to that stimulus. Within blocks of trials, the stimuli either follow a fixed sequence or are selected randomly. Learning is inferred by the difference in response latency between the sequence and random blocks (Cohen et al., 1990; Nissen and Buellemer, 1987). Using PET, neural regions showing regional cerebral blood flow ( $\mathrm{rCBF}$ ) changes related to sequence encoding were identified in two experiments in which the stimuli were cued by location (Grafton et al., 1995) or color (Hazeltine et al., 1997). In both studies, the task was first performed while subjects concurrently performed a secondary tone-

\footnotetext{
Received April 8, 1998; revised Aug. 21, 1998; accepted Aug. 24, 1998.

This work was supported in part by United States Public Health Service Grants NS 01568 and NS 33504 to S.T.G. and NS 30256 to R.B.I.

Correspondence should be addressed to Dr. Scott T. Grafton, Department of Neurology, Emory University School of Medicine, Woodruff Medical Research Building, Suite 6000, 1639 Pierce Drive, Atlanta, GA 30322.

Copyright (C) 1998 Society for Neuroscience $0270-6474 / 98 / 189420-09 \$ 05.00 / 0$
}

including sensorimotor cortex, supplementary motor area, and rostral inferior parietal cortex. After transfer, activity in inferior parietal cortex remained high, suggesting that this area had encoded the sequence at an abstract level independent of the particular effectors used to perform the task. In contrast, activity in sensorimotor cortex shifted to a more dorsal locus, consistent with motor cortex somatotopy. Thus, activity here was effector-specific. An increase in rCBF was also observed in the cingulate motor area at transfer, suggesting a role linking the abstract sequential representations with the task-relevant effector system. These results highlight a network of areas involved in sequence encoding and retrieval.

Key words: motor learning; human; sequencing; functional imaging; somatotopy; emission computed tomography; motor control

counting task that prevented awareness of the sequence. Consistent with previous studies of implicit learning, rCBF changes were mostly in motor areas, including the sensorimotor cortex, supplementary motor area (SMA), premotor cortex, and basal ganglia. This pattern was similar for both the location and color studies.

A puzzle presented by these results is that while implicit learning of a series of responses appears to be predominately supported by motor regions, behavioral evidence shows near perfect transfer of knowledge to novel sets of effectors. For instance, Keele et al. (1995) had subjects perform the SRT task under dual task conditions using either the four fingers of their right hands or a single finger. After several blocks of training, subjects switched their response technique. Reaction time benefits were nearly identical to those obtained from control subjects who responded in the same manner throughout the experiment (Cohen et al., 1990). In pilot experiments we too found excellent transfer between sequences learned while subjects made individuated finger responses on a small keyboard and responses made on a large keyboard that required movements of the entire arm.

It is tempting to infer that blood flow changes in the motor regions reflect an alteration of the limb representation (Grafton et al., 1992; Karni et al., 1995). However, such an interpretation does not provide a parsimonious explanation for the transfer of sequence knowledge to different effector groups. As subjects acquire a sequential motor skill with the SRT task, they could be learning any combination of at least three functional attributes: (1) the particular movements, linked to specific muscle groups (motor knowledge); (2) the sequence of stimuli instructing movements (perceptual knowledge); and (3) a more abstract level of 


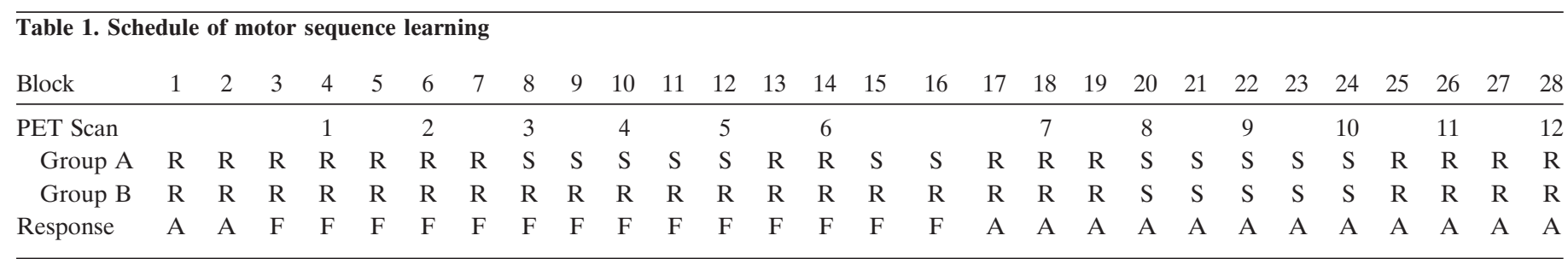

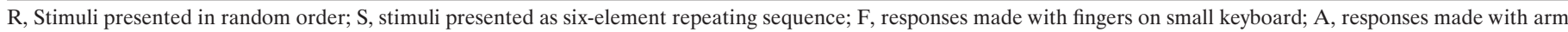
on large keyboard.

representation specifying a series of response (goal knowledge). Behavioral studies (Prinz and Nattkemper, 1986; Keele et al., 1995; Willingham, 1998) assessing transfer to novel series of stimuli and responses have favored the more flexible, goal-based sequence representation (MacKay, 1982). Thus, sequence knowledge appears to involve a representation of relatively abstract response goals, rather than being tied to a sequence of specific movements or gestures to particular locations.

The phenomenon of motor transfer provides an opportunity to evaluate the specific role of particular motor areas in sequence representation. In the current study, we independently manipulated the presence or absence of the sequence, and the manner in which subjects responded using a transfer paradigm. We sought to determine whether learning-related changes in $\mathrm{rCBF}$ were related to the specific effectors, a reflection of abstract motor goals, or some combination of these factors

\section{MATERIALS AND METHODS}

Subjects. Twenty normal young adult subjects (11 men and 9 women) volunteered for this study under informed consent in accordance with the Emory University Human Investigations Committee. Subjects were judged to be normal by excluding any previous neurological, psychiatric, or major medical history, and no person was on psychoactive medications. All subjects were strongly right-handed (Oldfield, 1971). The subjects were randomly assigned to one of two experimental groups (A and B). The mean age was 27.9 years in group A and 32.7 years in group B. These differences were not significant.

Behavioral tasks and performance measures. A modified version of the serial reaction time task was used to study longitudinal changes of $\mathrm{rCBF}$ during acquisition of a motor sequence (Hazeltine et al., 1997). Throughout the experiment a set of colored stimuli were used as instructional cues. The circular stimuli subtended $\sim 1^{\circ}$ of visual angle and were presented serially in the center of a computer monitor to eliminate sequential eye movements. The color of the stimuli (red, green, blue, or yellow) indicated which of four keys to press on a keyboard. In the first half of the experiment all 20 subjects used a small keyboard with four keys (interkey distance $2.7 \mathrm{~cm}$ ) to make discrete finger movements with the right hand, based on the four instructional stimuli. In the second half of the experiment, the subjects made motor responses with a large keyboard (interkey distance $20.7 \mathrm{~cm}$ ). They were told to hold the fingers of the right hand together to minimize distal finger movements. Thus, the two keyboards forced subjects to use predominately distal individuated finger muscles versus proximal arm muscles to make appropriate responses. The interstimulus interval was fixed at $1500 \mathrm{msec}$ with the circles visible for only the first $1000 \mathrm{msec}$. A block of trials consisted of 84 stimuli responses. Each block consisted of stimuli in random order or a repeating six-element sequence such as red, blue, red, yellow, blue, green.

To prevent the development of awareness of the sequence, subjects were required to perform a concurrent secondary task throughout the experiment. Subjects counted the number of $50 \mathrm{msec}$ low-pitched (200 $\mathrm{Hz}$ ) tones mixed randomly with high-pitched tones $(1000 \mathrm{~Hz})$. The presentation of the visual and auditory stimuli was made asynchronous by varying the delay between the onset of a colored circle and the onset of the tones by intervals of 1100,1200 , or $1300 \mathrm{msec}$. Between 50 and $75 \%$ of the tones were low-pitched targets. Dependent variables to assess performance on the SRT task included accuracy of motor response, response time (RT) (consisting of reaction time and movement time), and tone-counting accuracy. Our primary focus for evaluating learning was to compare the average RTs on sequence blocks compared with random blocks.

The presentation of sequential and random blocks followed the schedule shown in Table 1 . Subjects in group A learned the repeating sixelement sequence while using the small keyboard during blocks 8-12 and 15-16. Blocks 13 and 14 were random, allowing an initial assessment of sequence learning. After transferring to the large keyboard on block 17, the subjects completed three random blocks. Then, the sequence that was previously learned was reintroduced on block 20. Group A subjects then completed additional practice with the same sequence over blocks 20-24. Blocks 25-28 were random, allowing a second assessment of learning. Group B subjects completed only random blocks during the first half of the experiment, while using only the small keyboard. After transfer to the large keyboard, the six-element sequence was introduced for the first time on block 20 and continued through block 24. As with group A, sequence learning during performance with the large keyboard was assessed by the transition to random events on blocks 25-28. Assessment of sequence learning was made with repeated measures ANOVA. Within-group changes during sequence blocks $8-12$ as well as grouptask interactions over the same blocks were examined.

Imaging. Images of regional cerebral blood flow were determined using the PET autoradiographic method (Herscovitch et al., 1983; Raichle et al., 1983). For each scan, a bolus of $25 \mathrm{mCi}$ of $\mathrm{H}_{2}{ }^{15} \mathrm{O}$ was injected intravenously commensurate with the start of the behavioral task. A 90 sec scan was acquired in "three-dimensional (3-D) septa retracted mode" beginning $10 \mathrm{sec}$ after tracer administration. Attenuation correction was based on a calculated method using boundaries defined separately on each emission scan coupled with a transmission scan of the PET headholder. After reconstruction by filtered back-projection, image resolution was $11.8 \mathrm{~mm}$ full width at half maximum (FWHM) as verified by a line source. Blood samples were not acquired. Images of radioactive counts were used to estimate rCBF as described previously (Fox et al., 1984; Mazziotta et al., 1985).

Images of individual subject brain anatomy were determined with a high-resolution magnetic resonance imaging (MRI) scan. MRI scans were acquired on a 1.5 Tesla Philips Gyroscan NT scanner. A T-1 weighted 3-D fast field echo pulse sequence of 160 contiguous $1.3 \mathrm{~mm}$ coronal sections was obtained (TR/TE/flip angle $=33 \mathrm{msec} / 12 \mathrm{msec} /$ $35^{\circ}$ ). One subject could not complete the MRI because of claustrophobia.

Image analysis. For each subject, all PET scans were mutually coregistered to each other, and the mean PET was then coregistered to the same subject's MRI using an automated algorithm with error $<1 \mathrm{~mm}$ (Woods et al., 1998a). The MRIs from each subject were then coregistered using affine and then nonlinear algorithms to an MRI target atlas centered and rescaled to the Talairach atlas (Talairach and Tournoux, 1988; Woods et al., 1998b). The target was comprised of MRIs from 20 normal adult subjects. The MRI transformation matrices were then combined with the within-subject matrices to compute a direct transformation of the PET data to the atlas.

All PET studies were smoothed with a Gaussian filter to a final image resolution of $14.8 \mathrm{~mm} \mathrm{FWHM}$ and globally normalized to each other by proportional rescaling. Application of the general linear model of ANOVA were used to calculate task differences on a pixel by pixel basis without global pooling of image variance (Woods et al., 1996).

For the given experimental paradigm there were several possible approaches for identifying learning-related changes of brain activity. We used a simple model of learning predicated on the notion that areas involved in the initial encoding of a sequence should demonstrate progressive increases of brain activity. This can be tested with a repeated measures ANOVA design. Because there were only three scans acquired during presentation of the sequence in group A (e.g., blocks 8, 10, and 
Table 2. Localization of sequence encoding with a small keyboard

\begin{tabular}{|c|c|c|c|c|c|c|c|}
\hline \multirow[b]{2}{*}{ Anatomic location } & \multicolumn{3}{|c|}{$\begin{array}{l}\text { Talairach coordinates } \\
(\mathrm{mm})\end{array}$} & \multicolumn{3}{|c|}{$\begin{array}{l}\text { Group A } \\
\text { Mean rCBF (SD) }\end{array}$} & \multirow{2}{*}{$\begin{array}{l}\text { Uncorrected } \\
p \text { value } \\
\text { RM } \\
\text { ANOVA }\end{array}$} \\
\hline & $\mathrm{X}$ & $\mathrm{Y}$ & $\mathrm{Z}$ & Sequence 1 & Sequence 2 & Sequence 3 & \\
\hline L VL thalamus & -10 & -12 & 4 & $61.1(3.2)$ & $63.8(3.8)$ & $64.2(3.3)$ & $0.005^{*}, * *$ \\
\hline L postcentral sulcus $(40)$ & -50 & -11 & 28 & $59.9(4.8)$ & $60.8(3.0)$ & $62.1(4.2)$ & $0.005^{* * *}$ \\
\hline $\mathrm{L}$ angular gyrus (39) & -39 & -63 & 33 & $56.5(4.7)$ & $57.8(4.2)$ & $58.3(4.3)$ & 0.005 \\
\hline $\mathrm{L}$ anterior cingulate (32) & -14 & 21 & 41 & $57.7(3.2)$ & $61.1(4.2)$ & $61.4(2.8)$ & $0.005^{*}, * * *$ \\
\hline L cingulate gyrus (24/23) & -1 & -28 & 33 & $61.0(4.6)$ & $63.4(5.0)$ & $63.0(5.3)$ & $0.01 * *$ \\
\hline L superior parietal lobule (7) & -21 & -49 & 52 & $53.6(3.4)$ & $54.6(2.5)$ & $54.8(3.9)$ & $0.005^{*}$ \\
\hline L central sulcus $(4,3,1,2)$ & -20 & -23 & 57 & $51.3(2.9)$ & $52.8(3.3)$ & $53.1(3.0)$ & 0.01 \\
\hline R dorsal frontal gyrus (6) "SMA" & 4 & -5 & 67 & $66.8(4.8)$ & $69.6(5.4)$ & $69.6(6.8)$ & $0.01 * *$ \\
\hline
\end{tabular}

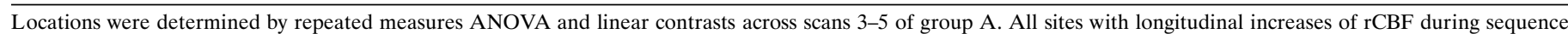

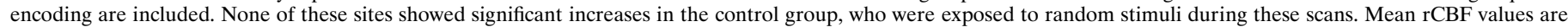
in units of milliliters per minute/100 gm of tissue. Anatomic locations in parentheses are Brodmann's areas according to the atlas of Talairach and Tournoux (1988).

* Significant at $p<0.05$ after correcting for multiple comparisons.

**Significant group-task interaction $(p<0.05)$.

$* * *$ Significant group-task interaction $(p<0.01)$.

12) the number of testable learning models was limited to monotonic changes (progressive increasing or decreases of activity over time) or quadratic changes. Quadratic changes were not tested, because their biological relevancy is unknown for this type of learning. None of the areas so defined showed within-group increases of activity for group B where only random blocks were presented. To minimize both Type I and Type II error, a pre-specified search volume smaller than the whole brain gray matter volume was used for these pixel by pixel calculations. From our previous studies of sequence learning using this task, we had a strong a priori prediction that $\mathrm{rCBF}$ changes would occur in motor areas located in the mesial wall and lateral precentral areas contralateral to the performing hand. The search volume covered the premotor, perisylvian, and parietal cortex extending posteriorly to the parieto-occiptial junction of the left hemisphere and bilateral mesial dorsal frontal and cingulate cortex. Bilateral dorsal medial and cingulate cortex were included to assure detection of blood flow changes close to the midline. This volume was defined manually, before the image analysis and generation of t-maps. Uncorrected significance for each site is listed in Table 2. Each region identified by the within-group repeated measures ANOVA of group A was further tested for significance after correcting for multiple comparisons using the method of Friston et al. (1994).

The statistical model defining sequence learning could be further constrained by also requiring greater activity in the sequence scans than the bracketing random blocks (scans 2 and 6). This more restrictive model was not used because it would only identify areas that can rapidly "deactivate" in the switch back to a random block. From our previous studies of sequence learning as well as those of Berns et al. (1997), there are multiple frontal cortical areas that don't display this property. Therefore, we did not use this additional constraint.

The statistical model could also be constrained by only including sites showing significant increases of activity in group A and not group B. This was determined by a group-task interaction term, calculated pixel by pixel and regionally. Without a very large number of subjects the pixel by pixel analysis of between-group differences is subject to both type I and type II errors (Woods, 1996). Therefore, we also tested each group A site showing a significant within increase of activity established by a withingroup repeated measures ANOVA, as described above. To do this, a group-task interaction term, testing for group A specific learning relative to group B, was calculated at each site. Those showing significant grouptask interactions are identified in Table 2.

The other purpose of the given experimental design was to identify sites representing a sequence irrespective of the effector used to perform the task. Such sites should show significant increases of activity after initial learning and also be increased in activity after transfer when the sequence was reintroduced at PET scan 8. To calculate the latter, repeated measures ANOVA was calculated within-group and further assessed by a regional group-task interaction effect.

Finally, a secondary analysis was performed to determine whether there were correlations between changes of response times during learning and changes of rCBF between pairs of sequence blocks. These were calculated separately for each region and group using a Pearson's correlation coefficient.

\section{RESULTS}

\section{Behavior}

All 20 subjects were able to complete the tasks with $<5 \%$ tone counting and tapping errors per block. Median RTs, calculated for each block of trials, are shown for the two subject groups in Figure 1 (for clarity only the RT data from the blocks in which PET images were obtained are shown). Group A subjects demonstrated a significant improvement in RTs during presentation of sequence blocks $8-12$ when responding on the small keyboard $\left(F_{(4,32)}=2.671 ; p<0.05\right.$ repeated measures ANOVA, missing data in one subject) whereas there was no significant change in

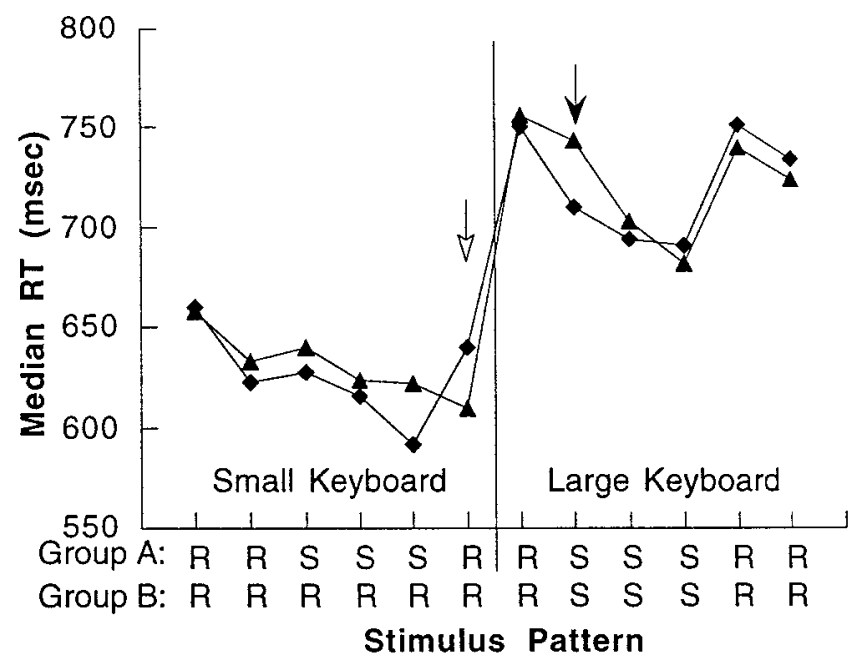

Figure 1. Changes of motor performance during acquisition of a motor sequence. Group A (diamonds) learned the sequence with a small keyboard. Sequence-specific learning is shown by the significant increase in RT when a random pattern is reintroduced (open arrow). After motor transfer (vertical line), responses were made with the large keyboard. A significant reduction of RT occurs when the previous sequence is reintroduced (solid arrow). For group B (triangles), there is no significant improvement of RT until the sequence is presented for the first time after transfer (solid arrow). 
group B $\left(F_{(4,36)}=1.284 ; p>0.29\right.$, repeated measures ANOVA). The primary behavioral test of learning is indicated by the open arrow in Figure 1. At the transition from block 12 to 13, the random sequence is reintroduced, and changes of performance should indicate sequence-specific learning rather than nonspecific effects. A test of group-task interaction by repeated measure ANOVA was significant for this transition $\left(F_{(1,17)}=5.679 ; p<\right.$ $0.029)$. As expected, group A subjects show a significant increase in RT at the transition to the random sequence $\left(t_{9}=3.622 ; p<\right.$ 0.006 , paired $t$ test) whereas there was no significant change in the performance of the group B subjects $\left(t_{9}=1.48 ; p>0.05\right.$, paired $t$ test). Further evidence of learning was assessed by reintroducing the already learned sequence during block 15 . There was a significant group-task interaction with reintroduction of the sequence in block $15\left(F_{(1,17)}=5.625 ; p<0.033\right.$, repeated measures ANOVA) related to large decrease of RT for group A and not group B. Together, these findings confirm that the behavioral changes observed in group A subjects were related to sequence learning during the first half of the experiment rather than a nonspecific time effect.

The second assay of learning is indicated by the solid arrow in Figure 1. Before this point, subjects have already completed three random blocks with the large keyboard. Note that there is a substantial increase in RT for both groups at transfer from block 16 to 17 , most likely caused by the fact that responses here required making large-scale movements. When the previously learned sequence is reintroduced during large keyboard movements at the transition from block 19 to 20, there is a significant group-task interaction by repeated measures ANOVA $\left(F_{(1,17)}=\right.$ 5.108; $p<0.037)$. Group A shows an immediate gain in performance with a significant reduction of RT $\left(t_{9}=3.91 ; p<0.004\right.$, paired $t$ test). In contrast, group B subjects, who have not been previously exposed to the sequence, show no improvement in RT $\left(t_{9}=0.34 ; p>0.05\right.$, paired $t$ test). Over the course of the ensuing sequence blocks 20-24 with the large keyboard, group B shows subsequent improvements of performance $\left(F_{(4,36)}=9.458 ; p<\right.$ 0.00002 , repeated measures ANOVA) whereas group A subjects, who have already learned the sequence, showed only a mild, nonsignificant reduction of RTs $\left(F_{(4,36)}=0.649 ; p>0.64\right.$, repeated measures ANOVA). Group-task interactions were significant confirming the greater learning in group B than group A $\left(F_{(4,68)}=3.22 ; p<0.018\right.$, repeated measures ANOVA). Evidence that performance-related changes for both groups was sequencespecific was corroborated by a significant increase of RTs when random stimuli were reintroduced in block 25: group $\mathrm{A}\left(t_{8}=\right.$ 6.468; $p<0.0002$, repeated measures ANOVA) and group B $\left(t_{9}=3.215 ; p<0.011\right.$, repeated measures ANOVA). In fact, at this last probe, learning was equivalent for the two groups as evidenced by the fact that the increase of RT is comparable $\left(F_{(1,17)}=1.96 ; p>0.17\right.$, repeated measures ANOVA).

It should be re-emphasized that the acquisition and transfer of sequential knowledge occurred implicitly in the current study. Consistent with previous studies (Nissen and Bullemer, 1987; Cohen et al., 1990; Grafton et al., 1995), the tone-counting task proved very effective in preventing awareness of the sequence. When probed at the end of the transfer phase, only one subject reported any awareness of the sequence. This subject was not included in the analysis.

\section{Imaging-motor sequence acquisition}

Sequence acquisition can be defined operationally as the time during which repeated exposure and practice of a sequential

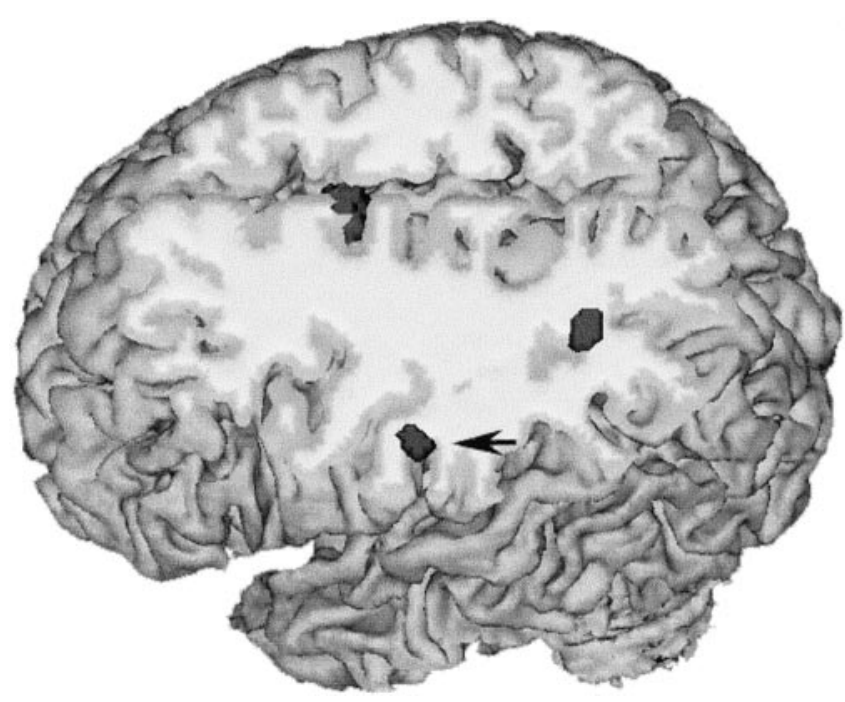

Figure 2. Cross-sectional anatomy of sequence encoding in parietal cortex. Parietal areas showing longitudinal increases of activity during sequence encoding in group A (across PET scans 3-5) are rendered in dark gray, superimposed over an individual subject's anatomic MRI. The site indicated with the black arrow is located in the postcentral gyrus, part of the rostral inferior parietal cortex (BA 40). The second site is in posterior parietal cortex in the left angular gyrus (BA 39). There is increasing activity in the interhemispheric fissure corresponding to the SMA (see Fig. 3 for details).

stimulus-response mapping leads to measurable improvements of performance. Acquisition occurred in group A when the sequence was presented and responses were made with the small keyboard and in group B when the sequence was introduced while responses were made with the large keyboard. From previous motor learning studies of the SRT task we predicted that sequence acquisition with the small keyboard would be accompanied by progressive increases of $\mathrm{rCBF}$ in several motor and perceptual areas, including primary sensorimotor cortex, supplementary motor area, and rostral inferior parietal cortex [Brodmann's area (BA) 40]. These predictions are substantiated in Table 2, which summarizes the location of these areas as well as increasing activity in posterior parietal, premotor, and anterior cingulate cortex contralateral to the hand performing the movements. The rostral and posterior parietal sites and mesial frontal area showing learning-related increases are shown in Figure 2. The results of the repeated measures ANOVA establish a relationship between mean changes of blood flow and mean reaction times for group A. No additional sites showing learning-specific increases in group A, but not group B, were identified when a pixel by pixel group-task interaction statistical image was calculated ( $p<0.005$ uncorrected). The data can also be queried to determine whether there is a more direct relationship between each individual subject's change of performance and change of regional blood flow. To do this, the percent increase of rCBF during presentation of the sequence blocks was correlated with the percent change of RT across individual subjects of group A for each of the sites in Table 2. The rostral inferior parietal area demonstrated a significant interaction between change of individual subject performance and change of $\operatorname{rCBF}(r=0.676$; $p<0.05)$.

The site in sensorimotor cortex was located $<9 \mathrm{~mm}$ from a site previously labeled as premotor cortex with the SRT task using color cues (Hazeltine et al., 1997). The position of this site, 

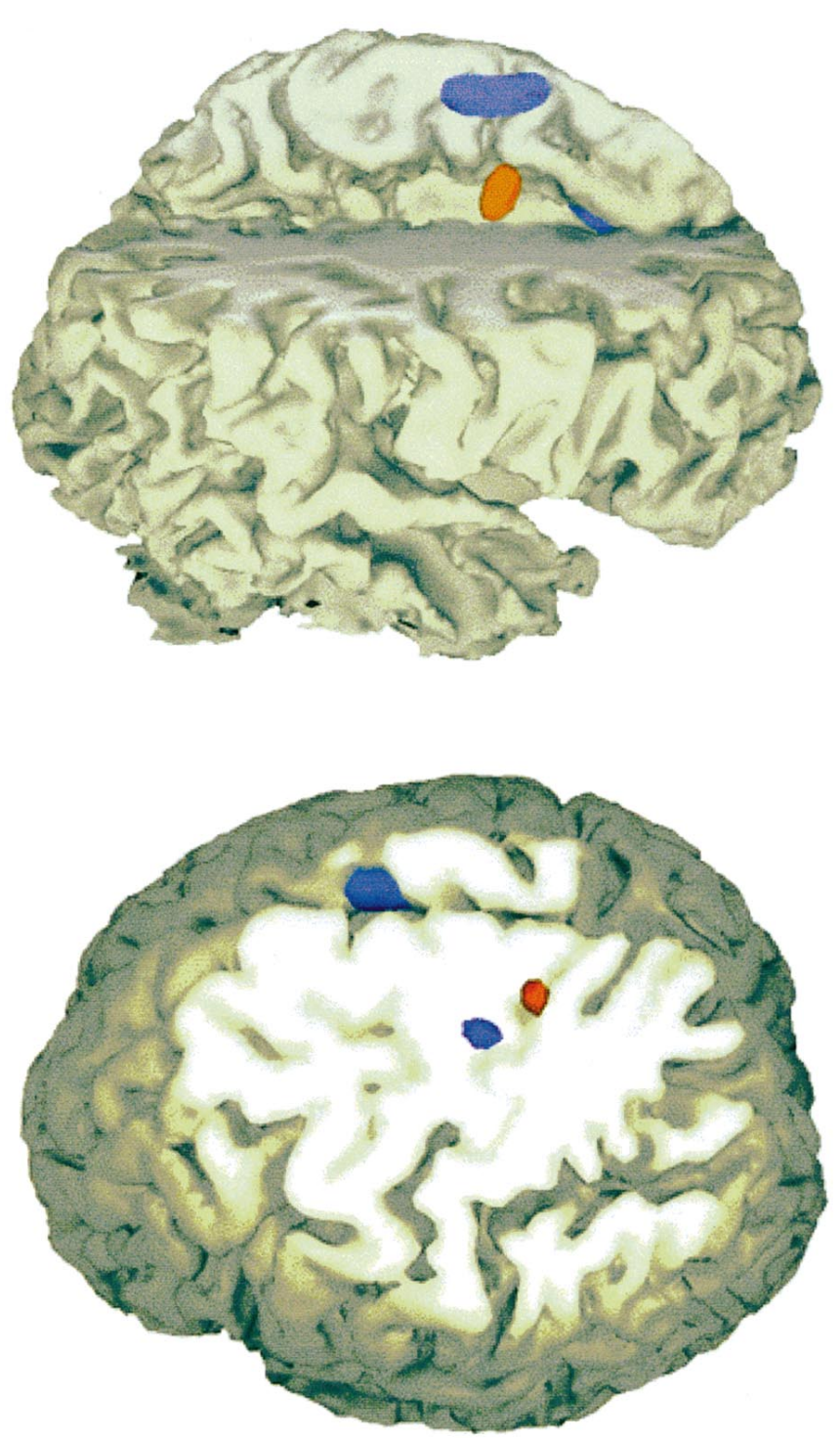

Figure 3. Cross-sectional anatomy of sequence encoding and retrieval. Areas showing longitudinal increases of activity during sequence encoding in group A (across PET scans 3-5) are rendered in blue, areas showing significant increases of activity when the previously learned sequence is reintroduced while performing with a new effector are shown in red. The results are superimposed over an individual subject's anatomic MRI. Sequence encoding with finger responses (blue) activates the SMA (top and bottom panels) and the primary sensorimotor cortex (bottom panel). Sequence retrieval while using whole-arm responses compared with randomly ordered stimuli and whole-arm responses (red) recruits the cingulate motor area (top panel) and a more superior-mesial site of the sensorimotor cortex (bottom panel). The shift in location within sensorimotor cortex demonstrates the dynamic and flexible localization of a sequence "representation" in sensorimotor cortex.

superimposed on gyral anatomy, is most consistent with a central sulcus/precentral gyrus location as shown in blue in Figure 3. The magnitudes of the group A regional blood flow increases, although significant, were weaker than what we observed in our previous two SRT learning studies. This is likely related to the fact that subjects were only trained with half as many blocks between PET scans on this study compared with previous experiments (to reduce the likelihood of developing fatigue).

During blocks 8-12, the group B subjects were presented with random events and as described above, did not show evidence of learning. As expected, rCBF changes in the learning-related sites for group A subjects were not significant for the group B subjects during this phase. In contrast, longitudinal decreases of activity in sensorimotor $(-27,-30,60)$ and posterior parietal cortex $(-25,-61$, 46) were observed for the group B subjects. After the introduction of the sequence during block 20 with the large keyboard, there was an early increase of activity in sensorimotor cortex followed by an increase in ventral premotor $(-40,0,21)$. Contrary to our expectations, we did not observe significant changes in rCBF in SMA, inferior parietal, or basal ganglia for this group as they acquired the sequence over blocks 20-24.

\section{Imaging-motor sequence retrieval}

Retrieval of a previously learned sequence could be examined by assessing for regional changes of rCBF between scans 7 (block 18) and 8 (block 20), indicated by the solid arrow in Figure 1 . Significant increases of activity in group A, but not group B, would indicate areas that are used to retrieve and execute a recently learned sequence. Sites showing significant increases of activity at this transition are summarized in Table 3.

There are three key observations from this comparison. First, there is a marked increase of activity in sensorimotor cortex when the sequence is reintroduced. The centroid of this $\mathrm{rCBF}$ increase is located $11 \mathrm{~mm}$ superior and caudal to the site associated with sequence learning using the small keyboard. The relative position of these two sites is shown in Figure 2. The difference in the location of the centroid is independent of the image resolution (they were independent image subtractions) and greater than what is observed in test-retest reproducibility experiments (Fox et al., 1987; Grafton et al., 1991). Given the large body of evidence demonstrating a crude somatotopy in motor cortex with proximal arm activity located superior to distal arm movements, the findings suggest that the locus related to sequence learning has shifted from distal to proximal limb areas of the motor cortex (Penfield and Boldrey, 1938; Woolsey et al., 1952; Colebatch et al., 1991; Walter et al., 1992; Grafton et al., 1993; Krings et al., 1997).

The second main result is the marked increase of activity in the dorsal cingulate cortex. The location of this site is in Brodmann's area 24, shown in red in Figure 3. The location is directly inferior to the supplementary motor area and is most likely within the dorsal cingulate motor area (He et al., 1995). The increase rCBF at this site is significantly greater when tested for a group-task interaction effect $\left(F_{(1,18)}=4.21 ; p<0.055\right)$. This test compares mean $\mathrm{CBF}$ changes between groups. We also found that individual subject improvements of RT for group A but not group B correlated with increases of $\mathrm{rCBF}$ at this location $(r=0.689 ; p<$ $0.03)$.

The third main result is a significant increase of $\mathrm{rCBF}$ in the rostral parietal cortex (BA 40) at the time of sequence retrieval. This same site demonstrated increasing activity with sequence encoding as well, and the centroid of this activation did not change location to the extent that the motor cortex site moved, suggesting rostral parietal cortex is involved in sequence representation irrespective of the motor effector.

Each of the cortical areas defined in the encoding or retrieval process demonstrated a distinct temporal profile of regional activity. Figure 4 presents the mean $\mathrm{rCBF}$ at identified sites for the group A subjects over the course of the sequence blocks and the neighboring random blocks. As in previous studies (Grafton et al., 1995; Karni et al., 1995; Hazeltine et al., 1997), motor cortex activity measured in the hand area showed longitudinal increases 
Table 3. Localization of sequence retrieval after motor transfer

\begin{tabular}{|c|c|c|c|c|c|c|c|}
\hline \multirow[b]{3}{*}{ Anatomic location } & \multicolumn{4}{|c|}{ Mean rCBF (SD) } & & & \\
\hline & \multicolumn{3}{|c|}{ Talairach coordinates $(\mathrm{mm})$} & \multicolumn{2}{|c|}{ Prior training } & \multicolumn{2}{|c|}{ Control group } \\
\hline & $\mathrm{X}$ & $\mathrm{Y}$ & $\mathrm{Z}$ & Random & Sequence & Random & Sequence \\
\hline L postcentral sulcus (40) & -55 & -16 & 31 & $64.8(3.2)$ & $66.3(4.0)$ & $66.6(6.1)$ & $67.0(6.4)$ \\
\hline L inferior parietal lobule $(40)$ & -31 & -52 & 40 & $55.3(5.1)$ & $56.6(5.2)$ & $58.9(8.5)$ & $60.5(8.7)$ \\
\hline $\mathrm{L}$ anterior cingulate $(24)^{*}$ & -3 & -3 & 45 & $69.4(3.6)$ & $72.8(4.1)$ & $70.2(5.6)$ & $69.5(5.0)$ \\
\hline L central sulcus $(4,3,1,2)$ & -16 & -31 & 64 & $58.9(3.9)$ & $61.3(4.2)$ & $61.3(4.8)$ & $62.0(5.0)$ \\
\hline
\end{tabular}

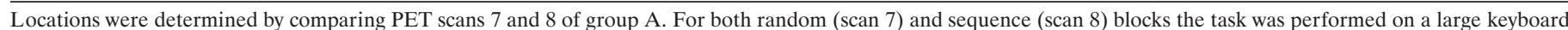

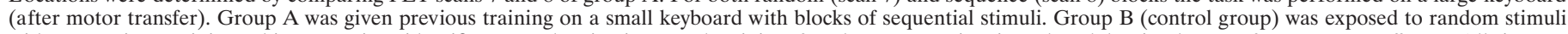

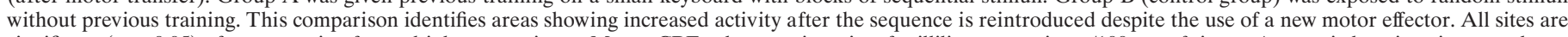

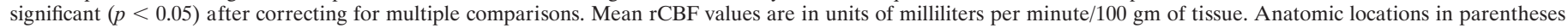
are Brodmann's areas according to the atlas of Talairach and Tournoux (1988).

*Significant group-task interaction $(p<0.05)$.

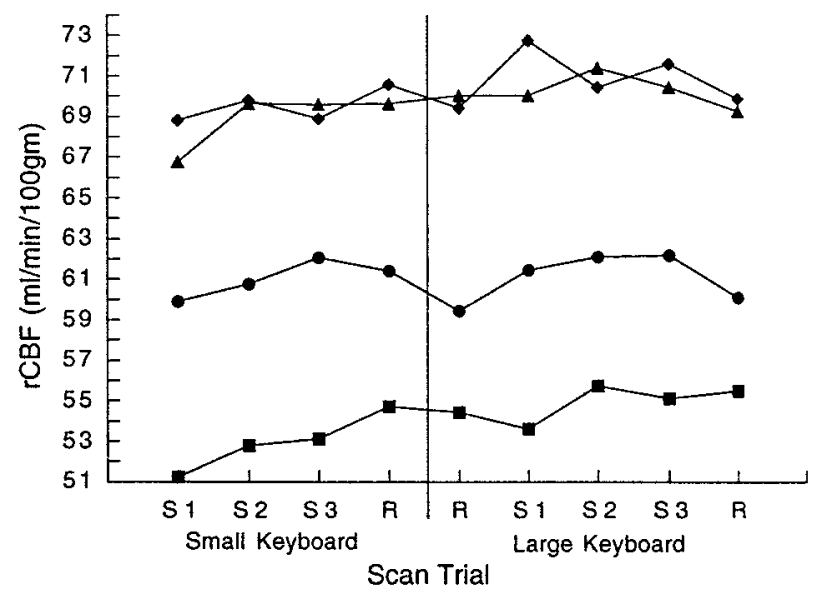

Figure 4. Changes of blood flow during sequence encoding and retrieval in group A subjects. After the initial presentation of a repeating sequence and responses made with the fingers on a small keyboard there is a progressive increase of activity in the inferolateral hand area of the sensorimotor cortex (squares, Talairach coordinates -20, -23, 57; Table 2). This increase plateaus at the time of transfer, and subsequent increases are not significant when the subjects respond with proximal arm movements and the large keyboard. Activity in the more mesial-rostral area of the sensorimotor cortex (Talairach coordinates -16, -31, 64; Table 3) demonstrate no change of activity until the sequence is reintroduced after transfer. In contrast, the SMA (triangles, Talairach coordinates 4, -5, 67) shows early increases of activity that do not change significantly for the remainder of the experiment, suggesting effector independence and an abstract representation of the sequence. The rostral parietal cortex (circles, Talairach coordinates $-50,-11,28$ ) shows a close correspondence to sequence encoding with longitudinal increases of activity during initial learning, then modulation linked to the presence or absence of the sequential or randomly ordered stimuli. The cingulate motor area (diamonds, Talairach coordinates -3, -3, 45; Table 3) showed a large jump of activity when the previously encoded sequence was reintroduced while subjects responded with a new effector (whole-arm movements). Note that this jump could not be secondary to kinematic differences of finger and arm movements, reflected in differences in the previous random to random transition (represented as a vertical line). The result suggests that the cingulate motor area may mediate transfer of sequential knowledge to different motor outputs.

with initial learning using the small keyboard. Activity in the more rostral arm area began to increase after transfer and with the reintroduction of the previously encoded sequence. In the supplementary motor area, activity increased early in the course of learning and remained elevated throughout the remainder of the experiment. SMA activity did not drop with presentation of random stimuli. In contrast, the rostral parietal cortex showed initial increases with learning. Subsequent increases were closely linked to the presence or absence of sequential versus random stimuli. The cingulate motor area showed overall increased activity with learning and a large jump when the sequence had to be retrieved with the new effector. None of these patterns were present in the group B subjects.

\section{DISCUSSION}

The results of this experiment serve to differentiate the functional anatomy of implicit sequence learning in humans. Each of the principal areas identified in the experiment demonstrates a distinct temporal profile in terms of sensitivity to the sequence and the mode of response. These patterns, shown in Figure 4, suggest specific learning-related functions.

The rostral parietal cortex (BA 40) appears to be most closely tied to sequence encoding. Activity in this region was highly contingent on the presence of the sequence pattern for both the small and large keyboards. This property matches a key feature of the behavioral data, namely that sequence knowledge transfers to novel sets of effectors. Thus, area 40 may represent the sequential order of the responses at a relatively abstract level, one that is independent of the actual muscles used to respond. Interestingly, $\mathrm{rCBF}$ in this site inversely correlated best with RTs during sequence encoding. A similar inverse relationship between RT and activity in the homologous right parietal cortex was observed in a single task version of the SRT task involving a 12-element sequence (Berns et al., 1997). Other PET studies of the SRT task have also reported enhanced activity here associated with sequence knowledge (Jenkins et al., 1994; Grafton et al., 1995; Hazeltine et al., 1997). Furthermore, in a PET study of motor preparation, area 40 was the only site showing statistically reliable increases in activity when preview information was given about an upcoming response compared with when no information was given (Deiber et al., 1996). Thus, this region may be crucial for planning movements, but at a representational level that best corresponds to the goals of the action rather than specific movements.

The supplementary motor area or adjacent pre-SMA may perform a related function of representing sequences at an abstract level. The activation in the current experiment encompassed bilateral SMA with a right-sided predominance. We propose that the early, sustained increase observed in bilateral SMA proper is consistent with the maintenance of an internal model of the sequence that is then used for driving movements, irrespec- 
tive of effector or sequence complexity. The notion of the SMA linked to internally generated models of movement including sequences is strongly supported by psychophysical lesioning and imaging data (Passingham, 1993; Jenkins et al., 1994; Tanji, 1994). PET studies also identify early increases of activity in SMA proper area during the initial acquisition of implicit motor skills, including the SRT task under dual task conditions (Grafton et al., 1992, 1994, 1995; Hazeltine et al., 1997). For the case of explicit sequence retrieval, three PET studies show increased activity in SMA proper related to sequence execution in a general sense, but not to sequence complexity (Sadato et al., 1996; Boecker et al., 1998; Catalan et al., 1998). Instead, there is increasing activity in pre-SMA during explicit retrieval of sequences of greater complexity. Activity in SMA proper of our study was insensitive to the reintroduction of random stimuli. It may be that an internal model is slow to change when there are variations of expected stimuli. As shown previously, it can take considerable time for blood flow increases in frontal areas to return to baseline during "unlearning" of a sequential code known implicitly (Berns et al., 1997).

There were two anterior cingulate sites demonstrating significant changes in this experiment. During initial sequence encoding the rostral anterior cingulate area increased in activity. This area has been the focus of numerous studies of attentional processes and the coordination of behavior in complex tasks. In a review, Posner (1994) noted that rostral anterior cingulate activity increases when subjects are required to engage in demanding tasks requiring internal monitoring or when responding to novel situations. This rostral portion of the anterior cingulate has also been shown to be active during conditions of increased response competition (Carter et al., 1998). Over the relatively short sequence used in our study, novelty would have been decreasing in relative importance and response competition would remain constant. In contrast, subjects were generating knowledge of the sequence order (although they were unaware). This knowledge could be coupled to top-down, directed attention to the stimulus features. After the initial learning this site showed no additional changes of activity over the remainder of the experiment, including sequence retrieval.

Increased activity was observed in a second cingulate focus during sequence retrieval. It was located immediately inferior to the supplementary motor area and comprises the caudal part of Brodmann's area 24 (Paus et al., 1996), i.e., the cingulate motor area (CMA). In nonhuman primates this area projects to spinal motor neurons and to prefrontal, SMA, premotor, and primary motor cortex, establishing it as a somatic motor area (Pandya et al., 1981). Recent anatomic evidence from nonhuman primates shows that the CMA can be further subdivided into rostral, ventral, and dorsal sections (He et al., 1995), with the locus from the present study probably located in dorsal CMA. The center of cingulate activity in the current study is in agreement with the arm area identified by Paus et al. (1993).

Less is known of this area's functionality with respect to other motor areas. The anterior cingulate in general, (including rostral cingulate cortex and CMA) has been proposed to be critical for shifting between behavioral states. The strong connections among the cingulate motor area, prefrontal cortex, and limbic areas imply that the site is critical for transducing higher level behavioral goals or thoughts into actions (Brooks, 1990). In monkeys, lesions of the dorsal medial frontal lobe that include the dorsal bank of the cingulate can lead to impairments of selecting between actions (Chen et al., 1995; Thaler et al., 1995). Lesions to the caudal anterior cingulate cortex in man can lead to akinetic mutism, motor neglect, and impaired motor initiation. Seizures and electrical stimulation to this area can cause complex limb movements that are superimposed onto ongoing movement (Talairach et al., 1973; Devinsky et al., 1995).

PET and fMRI studies identify activations involving CMA during voluntary movement (Paus et al., 1993; Kawashima et al., 1996a; Matsumara et al., 1996; Weiller et al., 1996; Kertzman et al., 1997; Van Oostende et al., 1997) and response selection (Kawashima et al., 1996b). Self-initiated movements cause greater and more rostral activation than stimulus-triggered movements (Larsson et al., 1996; Wessel et al., 1997). A similar functional gradient is observed in nonhuman primates (Shima et al., 1991). One previous learning study identified changes in this area that would suggest a role in sequence retrieval; there was greater activity during performance of a highly learned sequence compared with random scans (Doyon et al., 1996). In a PET study, a site close to the one reported here showed greater activation during the selection of incompatible motor responses (Paus et al., 1993). The researchers concluded that CMA was "funneling" high level commands to the executing neural structures. This framework is consistent with our results, whereby motor function for the CMA links an internal representation of an abstract sequence (at the target level) with the workspace requirements (at the effector level).

It could also be argued that the reintroduction of the sequence might be viewed as a novel event, a process known to enhance activity in more rostral cingulate cortex when a learned sequence is switched to a new one, even when subjects are unaware of the transition (Berns et al., 1997). This may be true, even though, at an explicit level, the task remained consistent across the random and sequence blocks. However, an increase of activity in this area was not seen for any of the other sequence to random transitions that could also be considered as novel events. Cingulate motor area activity was linked primarily to the reintroduction of the sequence after transfer to the large keyboard. This pattern indicates that the region may be critical for directing sequence information to regions controlling the appropriate effector set.

As in previous experiments, we observed a progressive increase of activity in motor cortex as subjects acquired a new movement pattern (Grafton et al., 1992, 1994, 1995; Karni et al., 1995; Doyon et al., 1996; Hazeltine et al., 1997). The increase is unlikely to be related to kinematic differences as the frequency and type of movements remained constant across scans. The increases of activity we observed in motor cortex were similar to the SMA during initial learning. However, unlike SMA, the sequence representation in motor cortex was closely linked to the effector at transfer. After behavioral transfer to a different effector, the center of sequence-related activity shifted to a more superior position, consistent with the general somatotopy of sensorimotor cortex. An interesting feature of the transfer results was that the shift was evident in the first post-transfer block. This suggests that sequence representation, at least as determined by blood flow imaging, is a dynamic phenomenon that can move within motor cortex, depending on workspace or motor output requirements.

Presumably the shift in motor cortex arose through the interactions of areas representing the sequence at an abstract, goalbased level with areas linking this knowledge with task-relevant effectors. Based on the areas activated in the current study, we hypothesize that the representation of the sequence can be linked to SMA and inferior parietal while the CMA provides a chan- 
neling operation that helps link this abstract information with a particular effector system (Paus et al., 1993).

This hypothesis has implications for interpreting learningrelated changes in motor cortex. Activity in this area may not reflect the encoding of sequential information per se, but rather, result from priming from upstream neural circuits such as the SMA or inferior parietal cortex. When expectations about forthcoming responses can be generated, these regions may provide anticipatory inputs to the motor cortex. As in other imaging work reporting motor cortex activation (Karni et al., 1995; Grafton et al., 1995; Hazeltine et al., 1997), there were relatively long intervals between consecutive responses, conditions that would be expected to be ideal for this sort of priming. This hypothesis may appear to conflict with results showing long-term changes in the functional organization of motor cortex after protracted training periods (Jacobs and Donoghue, 1991; Pons et al., 1991; Nudo et al., 1996;). However, in the SRT task we used, the individual movements are simple, discrete, and very well-learned. Under conditions such as this, it is likely that learning primarily occurs at a level of abstract response, or goal selection, rather than involving changes in movement kinematics (MacKay, 1982).

Some potential limitations of the current data should be borne in mind. By using a reduced brain volume for subsequent data analysis, we minimized type II errors while maximizing statistical sensitivity. Nevertheless, it is possible that there are additional cortical areas outside of the search volume that contribute to the process of sequence retrieval. A second important concern is whether any of the rCBF changes were simply caused by nonspecific time effects. This potential problem was reduced although not entirely eliminated by the use of a second group of subjects who were presented with only random targets during the first half of the experiment. For many of the sites showing learning-related increases of activity, there was a significant group-task interaction establishing that these increases were related to learning rather than time. For the remainder of the sites it should be noted that intergroup comparisons of PET data carry increased type II error limiting the sensitivity of this test statistic.

\section{REFERENCES}

Berns GS, Cohen JD, Mintun MA (1997) Brain regions responsive to novelty in the absence of awareness. Science 276:1272-1275.

Boecker H, Dagher A, Ceballos-Baumann AO, Passingham RE, Samuel M, Friston KJ, Poline J, Dettmers C, Conrad B, Brooks DJ (1998) Role of the human rostral supplementary motor area and the basal ganglia in motor sequence control: investigations with H2 $15 \mathrm{O}$ PET. J Neurophysiol 79:1070-1080.

Brooks VB (1990) Limbic assistance in task-related use of motor skill. In: The principles of design and operation of the brain (Eccles JC, Creutzfeldt O, eds), pp 343-368. Berlin: Springer.

Carter CS, Braver TS, Barch DM, Botvinick MM, Noll D, Cohen JD (1998) Anterior cingulate cortex, error detection, and the online monitoring of performance. Science 280:747-749.

Catalan MJ, Honda M, Weeks RA, Cohen LG, Hallett M (1998) The functional neuroanatomy of simple and complex sequential finger movements: a PET study. Brain 121:253-264.

Chen Y-C, Thaler D, Nixon PD, Stern CE, Passingham RE (1995) The functions of the medial premotor cortex II. The timing and selection of learned movements. Exp Brain Res 102:461-473.

Cohen A, Ivry RI, Keele SW (1990) Attention and structure in sequence learning. J Exp Psychol Learn Mem Cogn 16:17-30.

Colebatch JG, Deiber M-P, Passingham RE, Friston KJ, Frackowiak RSJ (1991) Regional cerebral blood flow during voluntary arm and hand movements in human subjects. J Neurophysiol 65:1392-1401.

Deiber M-P, Ibañez V, Sadato N, Hallett M (1996) Cerebral structures participating in motor preparation in humans: a positron emission tomography study. J Neurophysiol 75:233-247.
Devinsky O, Morrell MJ, Vogt BA (1995) Contributions of anterior cingulate cortex to behaviour. Brain 118:279-306.

Doyon J, Owen AM, Petrides M, Sziklas V, Evans AC (1996) Functional anatomy of visuomotor skill learning in human subjects examined with positron emission tomography. Eur J Neurosci 8:637-648.

Fox PT, Mintun MA, Raichle ME, Herscovitch P (1984) A non-invasive approach to quantitative functional brain mapping with $\mathrm{H} 215 \mathrm{O}$ and positron emission tomography. J Cereb Blood Flow Metab 4:329-333.

Fox PT, Miezen FM, Allman JM, Van Essen DC, Raichle ME (1987) Retinotopic organization of human visual cortex mapped with positronemission tomography. J Neurosci 7:913-922.

Friston KJ, Frith CD, Passingham RE, Liddle PF, Frackowiak RSJ (1992) Motor practice and neurophysiological adaptation in the cerebellum: a positron emission tomography study. Proc R Soc Lond B Biol Sci 248:223-228.

Friston KJ, Worsley KJ, Frackowiak RSJ, Mazziotta JC, Evans AC (1994) Assessing the significance of focal activations using their spatial extent. Hum Brain Mapp 1:210-220.

Grafton ST, Woods RP, Mazziotta JC, Phelps ME (1991) Somatotopic mapping of the primary motor cortex in man: activation studies with cerebral blood flow and PET. J Neurophysiol 66:735-743.

Grafton ST, Mazziotta JC, Presty S, Friston KJ, Frackowiak RSJ, Phelps ME (1992) Functional anatomy of human procedural learning determined with regional cerebral blood flow and PET. J Neurosci $12: 2542-2548$.

Grafton ST, Woods RP, Mazziotta JC (1993) Within arm somatotopy in human motor areas determined by PET imaging of cerebral blood flow. Exp Brain Res 95:172-176.

Grafton ST, Woods RP, Tyszka JM (1994) Functional imaging of procedural motor learning: relating cerebral blood flow with individual subject performance. Hum Brain Mapp 1:221-234.

Grafton ST, Hazeltine E, Ivry R (1995) Functional anatomy of sequence learning in normal humans. J Cognit Neurosci 7:497-510.

Hazeltine RE, Grafton ST, Ivry R (1997) Attention and stimulus characteristics determine the locus of motor sequence encoding: a PET study. Brain 120:123-140.

He S-Q, Dum RP, Strick PL (1995) Topographic organization of corticospinal projections from the frontal lobe: motor areas on the medial surface of the hemisphere. J Neurosci 15:3284-3306.

Herscovitch P, Markham J, Raichle ME (1983) Brain blood flow measured with intravenous H215O. I. Theory and error analysis. J Nucl Med 24:782-789.

Jacobs KM, Donoghue JP (1991) Reshaping the cortical motor map by unmasking latent intracortical connections. Science 251:944-947.

Jenkins IH, Brooks DJ, Nixon PD, Frackowiak RSJ, Passingham RE (1994) Motor sequence learning: a study with positron emission tomography. J Neurosci 14:3775-3790.

Karni A, Meyer G, Jezzard P, Adams MM, Turner R, Ungerleider LG (1995) Functional MRI evidence for adult motor cortex plasticity during motor skill learning. Nature 377:155-157.

Kawashima R, Itoh H, Ono S, Satoh K, Furumoto S, Gotoh R, Koyama M, Yoshioka S, Takahashi T, Takahashi K, Yanagisawa T, Fukuda H (1996a) Changes in regional cerebral blood flow during self-paced arm and finger movements. A PET study. Brain Res 716:141-148.

Kawashima R, Satah K, Itoh H, Ono S, Furumoto S, Gotoh R, Koyama M, Yoshioka S, Takahashi T, Takahashi K, Yanagisawa T, Fukuda H (1996b) Functional anatomy of GO/NO-GO discrimination and response selection-a PET study in man. Brain Res 728:79-89.

Keele SW, Jennings P, Jones S, Caulton S, Caulton D, Cohen A (1995) On the modularity of sequence representation. J Mot Behav 27:17-30.

Kertzman C, Schwarz U, Zeffiro TA, Hallett M (1997) The role of posterior parietal cortex in visually guided reaching movements in humans. Exp Brain Res 114:170-183.

Krings T, Buchbinder BR, Butler WE, Chiappa KH, Jiang HJ, Cosgrove GR, Rosen BR (1997) Functional magnetic resonance imaging and transcranial magnetic stimulation: complementary approaches in the evaluation of cortical motor function. Neurology 48:1406-1416.

Larsson J, Gulyas B, Roland PE (1996) Cortical representation of selfpaced finger movement. NeuroReport 7:463-468.

MacKay DG (1982) The problems of flexibility, fluency, and speedaccuracy trade-off in skilled behavior. Psychol Rev 89:483-506.

Matsumara M, Kawashima R, Naito E, Satoh K, Takahashi T, Yanagisawa T, Fukuda H (1996) Changes in rCBF during grasping in humans examined by PET. NeuroReport 7:749-752.

Mayr U (1996) Spatial attention and implicit sequence learning: evi- 
dence for independent learning of spatial and nonspatial sequences. $\mathrm{J}$ Exp Psychol Learn Mem Cogn 22:350-364.

Mazziotta JC, Huang S-C, Phelps ME, Carson RE, MacDonald NS, Mahoney K (1985) A noninvasive positron computed tomography technique using oxygen-15-labeled water for the evaluation of neurobehavioral task batteries. J Cereb Blood Flow Metab 5:70-78.

Nissen MJ, Bullemer P (1987) Attentional requirements of learning: evidence from performance measures. Cogn Psychol 19:1-32.

Nudo RJ, Milliken GW, Jenkins WM, Merzenich MM (1996) Usedependent alterations of movement representations in primary motor cortex of adult squirrel monkeys. J Neurosci 16:785-807.

Oldfield RC (1971) The assessment and analysis of handedness: the Edinburgh inventory. Neuropsychologia 9:97-113.

Pandya DN, Van Hoessen GW, Mesulam MM (1981) Efferent connections of the cingulate gyrus in the rhesus monkey. Exp Brain Res 42:319-330.

Pascual-Leone A, Grafman J, Hallett M (1994) Modulation of cortical motor output maps during development of implicit and explicit knowledge. Science 263:1287-1289.

Passingham R (1993) The frontal lobes and voluntary action. Oxford: Oxford UP.

Paus T, Petrides M, Evans AC, Meyer E (1993) Role of the human anterior cingulate cortex in the control of oculomotor, manual, and speech responses: a positron emission tomography study. J Neurophysiol 70:453-469.

Paus T, Tomaiuolo F, Otaky N, MacDonald D, Petrides M, Atlas J, Morris R, Evans AD (1996) Human cingulate and paracingulate sulci: pattern, variability, asymmetry, and probabilistic map. Cereb Cortex 6:207-214.

Penfield W, Boldrey E (1938) Somatic motor and sensory representation in the cerebral cortex of man as studied by electrical stimulation. Brain 15:389-443.

Pons TP, Garraghty PE, Ommaya AK, Kaas JH, Taub E, Mishkin M (1991) Massive cortical reorganization after sensory deafferentation in adult macaques. Science 252:1857-1860.

Posner MI (1994) Attention: the mechanisms of consciousness. Proc Natl Acad Sci USA 91:7398-403.

Prinz W, Nattkemper D (1986) Effects of secondary tasks on search performance. Psychol Res 48:47-51.

Raichle ME, Martin WRW, Herscovitch P (1983) Brain blood flow measured with intravenous H215O. II. Implementation and validation. J Nucl Med 24:790-798.

Sadato, N, Campbell G, Ibanez R, Deiber M, Hallett M (1996) Complexity affects regional cerebral blood flow change during sequential finger movements. J Neurosci 16:2691-2700.

Schmidtke V, Heuer H (1996) Secondary-task effects on sequence learning. Psychol Res 59:119-133.

Seitz RJ, Roland PE (1992) Learning of sequential finger movements in man: a combined kinematic and positron emission tomography (PET) study. Eur J Neurosci 4:154-165.

Shadmehr R, Holcomb HH (1997) Neural correlates of motor memory consolidation. Science 277:821-825.

Shima K, Aya K, Mushiake H, Inase M, Aizawa H, Tanji J (1991) Two movement-related foci in the primate cingulate cortex observed in signal-triggered and self-paced forelimb movements. J Neurophysiol 65:188-202.

Talairach J, Tournoux P (1988) Co-planar stereotaxic atlas of the brain. New York: Thieme.

Talairach J, Bacaud J, Geier S, Bordas-Ferrer M, Bonis A, Sziklz G, Rusu M (1973) The cingulate gyrus and human behavior. Electroencephalogr Clin Neurophysiol 34:45-52.

Tanji J (1994) The supplementary motor area in the cerebral cortex. Neurosci Res 19:251-268.

Thaler D, Chen Y-C, Nixon PD, Stern CE, Passingham RE (1995) The functions of the medial premotor cortex I. Simple learned movements. Exp Brain Res 102:445-460.

Van Oostende S, Van Hecke P, Sunaert S, Nuttin B, Marchal G (1997) FMRI studies of the supplementary motor area and the premotor cortex. NeuroImage 6:181-190.

Walter H, Kristeva R, Knorr U, Schlaug G, Huang Y, Steinmetz H, Nebeling B, Herzog H, Seitz RJ (1992) Individual somatotopy of primary sensorimotor cortex revealed by intermodal matching of MEG, PET, and MRI. Brain Topogr 5:183-187.

Weiller C, Juptner M, Fellows S, Rijntjes M, Leonhardt G, Kiebel S, Muller S, Diener HC, Thilmann AF (1996) Brain representation of active and passive movements. NeuroImage 4:105-110.

Wessel K, Zeffiro T, Toro C, Hallett M (1997) Self-paced versus metronome-paced finger movements. J NeuroImage 7:145-151.

Willingham DB (1998) Implicit motor sequence learning is not purely perceptual. Mem Cognit, in press.

Woods RP (1996) Modeling for intergroup comparisons of imaging data. NeuroImage 4:S84-S94.

Woods RP, Iacoboni M, Grafton ST, Mazziotta JC (1996) Three-way analysis of variance. In: Quantification of brain function using PET (Myers R, Cunningham V, Bailey D, eds), pp 353-358. New York: Academic.

Woods RP, Grafton ST, Holmes CJ, Cherry SR, Mazziotta JC (1998a) Automated image registration: I. General methods and intrasubject validation. J Comput Assisted Tomogr 22:139-152.

Woods RP, Grafton ST, Watson JDG, Sicotte NL, Mazziotta JC (1998b) Automated image registration: II. Intersubject validation of linear and nonlinear models. J Comput Assisted Tomogr 22:155-165.

Woolsey CN, Settlage PH, Meyer DR, Sencer W, Hamuy TP, Travis AM (1952) Patterns of localization in precentral and "supplementary" motor areas and their relation to the concept of a premotor area. Res Publ Assoc Res Nerv Ment Dis 30:238-264. 